m09.p01

\section{Inhibitory Complexes of N10- formyltetrahydrofolate Synthetase Indicate Negative Cooperativity between Subunits} $\frac{\text { Magdalena Bielak }}{\text { Lukasz Lebioda }^{\mathrm{b}}}{ }^{\text {a }}$ Geqing Chai, ${ }^{\mathrm{b}}$ Krzysztof Lewinski ${ }^{\mathrm{a}}$,

${ }^{a}$ Faculty of Chemistry, Jagiellonian University, Cracow, Poland, and ${ }^{b}$ Department of Chemistry and Biochemistry, University of South Carolina, Columbia,USA.E-mail: bielak@chemia.uj.edu.pl

\section{Keywords: N10-formyltetrahydrofolate synthetase, cooperativity, folate enzymes}

$\mathrm{N}^{10}$-Formyltetrahydrofolate synthetase (FTHFS) catalyzes the formylation of tetrahydrofolate $\left(\mathrm{H}_{4}\right.$ folate $)$ in an ATP dependent reaction, an initial step in the reduction of carbon dioxide and other one-carbon precursors to acetate (and the reverse reaction). The enzyme, present at high levels in the acetogenic and purinolytic bacteria, is involved in a $\mathrm{C} 1$ carbon fixation process for cellular biosynthesis or in ATP synthesis.

Here, we report the crystal structures of two nonisomorphous, inhibitory complexes of FTHFS from Morella thermoacetica. The first, obtained at ca $2.0 \mathrm{M}$ ammonium sulfate, is with a catalytic intermediate - formylphosphate, obtained from ATP, and an additional molecule of ATP, which crowds the active site. This explains previously observed substrate inhibition [1]. The other structure is an inhibitory complex of FTHFS with antifolate ZD9331, which crystallized from PEG. Structures have been determined by molecular replacement with the structure of native FTHFS (pdb code: 1EG7) as the starting model and refined using CNS to R factors of 20.4 and 20.1, respectively.

The ligands positions allowed us to identify the active site. In both structures the aromatic moieties of ATP and ZD9331 are sandwiched between Phe384 and Trp412. The overlap of the ligands binding sites suggests a double displacement mechanism for FTHFS catalysis rather than the previously proposed random sequential mechanism [2].

FTHFS functions as a tetramer, loose dimer of tightly bound dimers. In both complexes (and in the native structure) the loose interaction corresponds to two-fold crystallographic symmetry. Tight dimmers are asymmetric and there is only one formylphosphate/ATP or ZD9331 per dimmer. Moreover, the complex asymmetry correlates with the crystal packing generating perfectly ordered systems; this is observed for both complexes despite very different crystal packing. The asymmetry of the complexes thus indicates strong negative cooperativity between the tight dimer subunits. An analysis of interactions that communicate ligand binding in subunit $A$ to subunit $B$ and prevent simultaneous ligand binding in subunit B will be presented.

[1] Himes, R. H., and Harmony, J. A. K. (1973) CRC Crit. Rev. Biochem. $1,501-535$.

[2] Leaphart, A., Spencer, H. T., and Lovell, C. R. Arch. Biochem. Biophys. (2002) 408, 137-43. m09.p02

\section{Structure of a novel acyltransferase}

$\underline{\text { R. Bott }}{ }^{1}$, M. Saldajeno ${ }^{1}$, G. Ganshaw ${ }^{1}$, M. Cervin ${ }^{1}$, Gregg Whited $^{1}$, W Weyler ${ }^{1}$, R. Sala ${ }^{1}$, M. Soltis ${ }^{2}$, M. Irimpan ${ }^{2}$

${ }^{1}$ Genencor International a Division of Danisco, Palo Alto California 94304 and ${ }^{2}$ SSRL, Stanford University, Stanford, CA 94309

Keywords: acyltransferase, SGBH Hydrolase, alcoholysis

We have determined the three dimensional structure of a unique acyltransferase that catalyzes acyl transfer reactions in water. Unlike hydrolases that perform alcoholysis under anhydrous conditions, this acyltransferase, isolated from Mycobacterium smegmatis (MsAcT), demonstrated alcoholysis even in substantially aqueous media. MsAct crystallizes as an octamer in the tetragonal space group P4. The molecule was determined using selenomet MAD phasing and has been refined to a working $\mathrm{R}$-factor of $17.5 \%$ and $\mathrm{R}_{\text {free }}$ of $19.6 \%$. A structural-homology search performed with MsAcT using the program DALI [1], which is based on a distance criterion and does not use sequence information for the comparison, showed five closely related proteins all belonging to the SGNH hydrolase class of enzymes. The structure of MsAct provides additional insight into the structural basis of its ability to perform acyl transfer in aqueous media.

[1] Holm, L. and Sander C. (1995) Dali: A network tool for protein structure comparisons, Trends Biochem. Sci. 478-480. 